\title{
Raman Spectroscopic Detection of Rapid, Reversible, Early-Stage Inflammatory Cytokine-Induced Apoptosis of Adult Hippocampal Progenitors/Stem Cells: A Preliminary Study
}

\author{
UMA LADIWALA ${ }^{1}$, ASEEFHALI BANKAPUR ${ }^{2}$, SUREKHA BARKUR ${ }^{2}$, BHUSHAN THAKUR ${ }^{1}$, \\ CHIDANGIL SANTHOSH ${ }^{2}$ and DEEPAK MATHUR ${ }^{1,3 *}$ \\ ${ }^{1}$ UM-DAE Centre for Excellence in Basic Sciences, Kalina Campus, Mumbai 400 098, India \\ ${ }^{2}$ Centre for Atomic and Molecular Physics, Manipal University, Manipal 576 104, India \\ ${ }^{3}$ Tata Institute of Fundamental Research, 1 Homi Bhabha Road, Mumbai 400 005, India
}

(Received on 5 May 2015; Revised on 14 August 2015; Accepted on 24 August 2015)

\begin{abstract}
The role of neuro-inflammation in diverse, acute and chronic brain pathologies is being increasingly recognized. Neuroinflammation is accompanied by increased levels of both pro- and anti-inflammatory cytokines; these have deleterious as well as protective/reparative effects. Inflammation has varying effects on neurogenesis and is a subject of intense contemporary interest.We show that TNF- $\alpha$ and IFN- $\gamma$, used concomitantly, cause apoptosis of adult rat hippocampal progenitor/stem cells in vitro as detected by the TUNEL and MTT assays on time scales of several hours. We have coupled Raman spectroscopy to an optical trap to probe early changes of apoptosis in single, live neural stem cells that have been treated with pro-inflammatory cytokines, TNF- $\alpha$ and IFN- $\gamma$. Our spectroscopic results on very early-stage apoptosis also show that cells can be divided into two groups: those that show an increase in the intensity of the DNA band and those that show the opposite. Changes caused by inflammation-induced denaturation of DNA are observed in the Raman spectra that correspond to very early stages of apoptosis, occurring on very fast time scales: as short as 10 minutes. Addition of the anti-inflammatory cytokine IL-10 either 10 min before or 10 min after treatment with TNF- $\alpha$ and IFN- $\gamma$ reverses the changes substantially. The findings of this preliminary study imply that inflammation may induce very rapid changes leading to cell death but that these are reversible, in the early stages at least.
\end{abstract}

Keywords: Neural Stem Cell; Raman Spectroscopy; Cytokine; Apoptosis

\section{Introduction}

Brain inflammation is known to be a complex cascade of cellular and molecular responses to stress, injury, infection or neuro degeneration of the central nervous system (CNS). The inflammatory response, which attempts to get rid of, or contain, the stressor and return the CNS to its normal state, occurs within minutes and involves both cells as well as an array of immune mediators like the cytokines.

A commonly observed outcome of neuroinflammation is apoptosis, that is, cell death of neural cells, which is characterized by DNA condensation and fragmentation, nuclear shrinkage, chromatin and cytoplasmic condensation and disintegration (Kerr et al., 1972; Wyllie et al., 1980). It appears that cells in the hippocampus, one of the regions of ongoing neurogenesis, are particularly vulnerable to both acute and chronic inflammation as occur in traumatic brain injury (TBI) and neurodegenerative disorders. The cognitive deficit with memory impairment and emotional disturbances that is often a sequel to brain inflammation, is due both to neuronal apoptosis as well as damage to neural stem cells (NSCs) with disrupted

*Author for Correspondence: E-mail: atmol1@tifr.res.in; Tel: 02222782736 
neurogenesis in the hippocampus (Krawohl and Kaiser, 2004a,b; Peng et al., 2008; Waldau and Shetty, 2008). Acute inflammation is accompanied by increased levels of pro-inflammatory cytokines such as TNF- $\alpha$, IL-1 $\beta$, IL-6 and IFN- $\gamma$ (Csuka et al., 1999; Morganti-Kossman et al., 2001; Goodman et al., 2008; Frugieret al., 2010; Dalgard et al., 2012; Christie and Turnley, 2013) which have varied effects on neurogenesis (Ben-Hur et al., 2003; Wong et al., 2004; Cacci et al., 2005; Sheng et al., 2005; Iosif et al., 2006; Tzong-Shine et al., 2008). Acute insult also raises levels of anti-inflammatory cytokines like IL10 (Csuka et al., 1999; Hensler et al., 2000; Dziurdzik et al., 2004; Shiozaki et al., 2005; Kamm et al., 2006; Kirchoff et al., 2008).

The injured brain attempts repair by producing new neurons. Injury is also amenable to rescue by endogenous or exogenous therapeutic interventions such as cell transplantation. For such interventions to succeed, it is crucial to identify very early time points at which changes of cell death or damage occur postinjury. It has hitherto been observed, using standard biological assays that such changes occur on time scales of several hours to days (Conti et al., 1998; Holmin and Hojeberg, 2004; Sheng et al., 2005; Cacci et al., 2005). In experiments whose results we report in the following we have made use of microRaman spectroscopy (RS) coupled to an optical trap to detect and quantify early changes of apoptosis in live neural stem cells that have been treated with proinflammatory cytokines, TNF- $\alpha$ and IFN- $\gamma$. Our methodology is such that we are able to detect, for the first time,the effect of pro-and anti-inflammatory cytokines on neural stem cells on time scales as short as 10 minutes. Our spectroscopic results on very earlystage apoptosis also show that cells can be divided into two groups: those that show an increase in the intensity of the DNA band and those that show the opposite. We observe that addition of the antiinflammatory cytokine IL-10 either $10 \mathrm{~min}$ before or 10 min after treatment with TNF- $\alpha$ and IFN$\gamma$ reverses the changes substantially. The findings of our preliminary set of experiments open new vistas for studies than can probe, in detail, the extent to which inflammation can induce very rapid changes leading to cell death. Our work indicates that such rapid changes are reversible at the early time points tested.

\section{Methods}

\section{Single Cell Spectroscopy with Raman Tweezers}

Raman spectroscopy measurements were carried out using a home built single-beam Raman Tweezers setup whose details have been published elsewhere (Bankapur et al., 2010; Zachariah et al., 2010). A schematic representation is shown in Fig. 1A along with a typical Raman spectrum of the NSC (Fig. 1B) recorded by keeping a slit width of $100 \mu \mathrm{m}$. The Raman spectrum was measured using a single laser beam (785 nm wavelength) to optically trap, and excite, a single live cell for Raman scattering. The spatial intensity distribution within the laser beam was expanded from nearly $1.5 \mathrm{~mm}$ in diameter (the size of the beam emanating from our diode laser) to 10 $\mathrm{mm}$ using a Galilean type beam expander comprising a plano-concave lens $(\mathrm{f}=5 \mathrm{~cm})$ and a biconvex lens $(\mathrm{f}=30 \mathrm{~cm})$. A dichroic mirror with high reflectivity at $785 \mathrm{~nm}$ and high transmission above $785 \mathrm{~nm}$ directed the beam vertically into the microscope objective of an inverted microscope (Nikon Eclipse Ti-U, Japan) such that the expanded beam overfilled the objective (Fig. 1A). The objective had a large numerical aperture (1.3 NA) and 100x magnification (Nikon, Japan) which enabled the laser beam to be focused to a near-diffraction limited spot, thus facilitating the formation of a high intensity gradient that is crucial for optical trapping of single cells (Ashkin, 1970).

Backscattered light was collected by the same microscope objective and was directed to the output port of the microscope by an optical path selector. The weak Raman signal was separated from the accompanying strong Rayleigh signal by means of an optical edge filter (Semrock, USA). The filtered Raman signal was focused onto the slit of an $f / 4.1$ spectrograph (iHR 320, Horiba JobinYvon, Japan) equipped with a holographic grating (1200 grooves/ $\mathrm{mm})$ which laterally dispersed the signal. A concave mirror $(\mathrm{f}=32 \mathrm{~cm})$ focused the laterally separated spectral lines (the Raman lines constituting the spectrum, as shown in Fig. 1B) on to a liquid nitrogen cooled CCD detector (Symphony, Horiba Jobin Yvon, Japan). We determined the resolution of our 

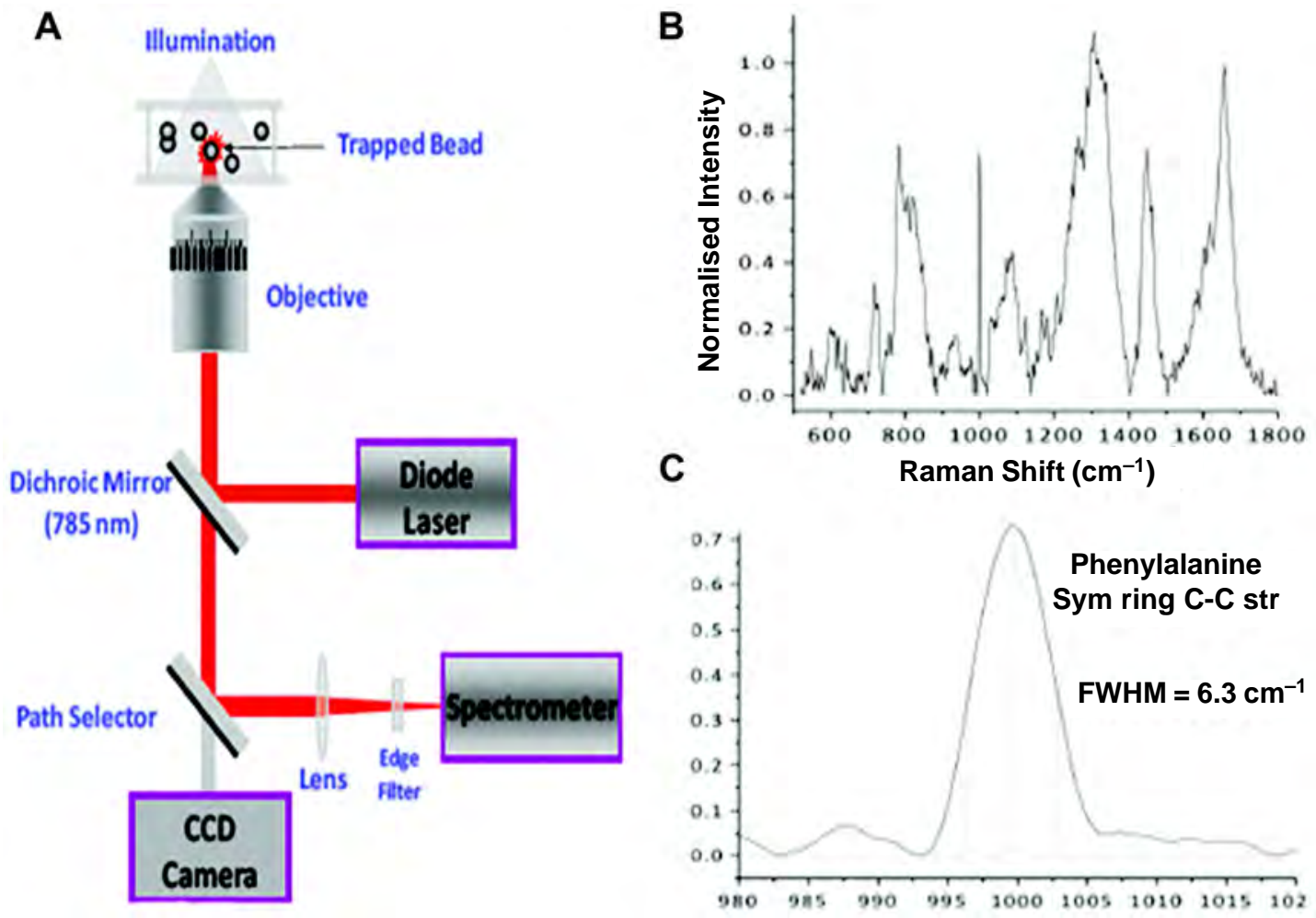

Fig. 1: A: Schematic representation of the single beam Raman tweezers set-up. B: Raman spectrum of a single, live cell. C: Determination of spectrometer resolution with respect to phenylalanine peak at $999 \mathrm{~cm}^{-1}$ in the above spectrum

spectrometer with respect to the phenylalanine peak at $999 \mathrm{~cm}^{-1}$ of the cell spectrum (Fig. 1C) to be 6.3 $\mathrm{cm}^{-1}$.

Our measurement procedure in the present series of experiments was as follows. Raman spectra were recorded by us using NSCs before and after treatment with the inflammatory cytokines TNF- $\alpha$ and IFN- $\gamma$, with and without the anti-inflammatory cytokine IL-10. Adult rat hippocampal NSCs/ progenitors were suspended in phosphate buffer saline (PBS). $200 \mu \mathrm{l}$ of this suspension was placed in a custom-made sample cell and control Raman spectra were acquired by trapping onecell from the suspension at a time. After acquisition of Raman data, $20 \mathrm{ng} / \mathrm{ml}$ each of TNF- $\alpha$ and IFN- $\gamma$ were added to this $200 \mu \mathrm{l}$ cell suspension. Care was taken to retain the cell from which the control Raman spectrum was recorded within the trapping volume while adding the cytokines. The trapped cell was left for 10 minutes under the effect of the added cytokines following which three sets of Raman spectra (10 minutes acquisition time in each case) were recorded consecutively from the same cell. The experiment was repeated by adding $20 \mathrm{ng} / \mathrm{ml}$ of IL-10, either 10 minute pre- or posttreatment with TNF- $\alpha+$ IFN- $\gamma(20 \mathrm{ng} / \mathrm{ml}$ each $)$. The three experimental conditions utilized in our series of measurements on different sets of cells from the same culture but over several consecutive days (identified as $\mathrm{x}, \mathrm{y}, \mathrm{z}, \ldots)$ are as summarized in Table 1.

To summarize, we recorded (a) four spectra each from a selected live, trapped cell in every condition, (b) one spectrum before adding the cytokines (baseline control), and (c) three successive spectra 10 minutes after addition of the cytokines. The results that we present encompass 32 spectral measurements from 8 neural stem cells. Control experiments were also performed by recording three successive Raman spectra every 10 minutes from two cells, without adding any of the cytokines.

It is pertinent to note that we subjected raw spectra acquired in our experiments to 5-point moving 
Table 1: Summary of the measurement procedure followed in the present experiments.

\begin{tabular}{llll}
\hline Condition & \multicolumn{2}{l}{ Cytokines added after recording baseline control spectrum } & Raman Spectra Recorded \\
& $0^{\text {th }}$ Minute & $10^{\text {th }}$ Minute & \\
\hline I & TNF- $\alpha+$ IFN- $\gamma$ & - & Control x, Cycle x.1, Cycle x.2, Cycle x.3 \\
II & IL-10 & TNF- $\alpha+$ IFN- $\gamma$ & Control y, Cycle y.1, Cycle y.2, Cycle y.3 \\
III & TNF- $\alpha+$ IFN- $\gamma$ & IL-10 & Control z, Cycle z.1, Cycle z.2, Cycle z.3 \\
\hline
\end{tabular}

averages smoothing and, subsequently, multi-point baseline correction was applied. Such baseline subtraction becomes necessary because fluorescence signals covering a wide range of frequencies inevitably accompany Raman spectra. We also acquired spectra bare glass slides so as to enable us to identify certain spectral features being contributed by the glass slide rather than the cells placed on the slide.

\section{Isolation of Hippocampal Progenitors/stem Cells, Cytokine Treatments, TUNEL and MTT assays}

Hippocampal progenitors/stem cells were isolated from Wistar rats (wt. 200-250 $\mathrm{gm}$ ) according to a modification of an earlier described protocol (Palmer et al., 1995). Briefly, hippocampi were dissected from brains of the rats and neural progenitor cells were isolated using a Percoll density gradient centrifugation. To obtain fairly pure populations of neural progenitors, the isolated cells were initially plated on poly-Lornithine $(20 \mu \mathrm{g} / \mathrm{ml})$ and laminin $(10 \mu \mathrm{g} / \mathrm{ml})$ - coated T-25 flasks in DMEM/F-12 medium supplemented with B-27 and 40ng/ml FGF-2 at $37^{\circ} \mathrm{C}$ in a humidified atmosphere with $5 \% \mathrm{CO}_{2}$. After about 3 weeks, dense colonies of proliferating progenitor-like cells were manually stripped, mechanically dissociated and plated in poly-L-ornithine-coated T-25 flasks (Nunc, USA) in Neurobasal medium supplemented with B-27 and $40 \mathrm{ng} / \mathrm{ml}$ FGF-2. NSCs were characterized by immunocytochemical detection of marker expression.

To ascertain the effects of cytokines on survival/ cell death of adult rat hippocampal progenitors in vitro, the TUNEL (Terminal deoxynuceotidyl transferase dUTP nick-end labelling) assay was performed using the TACS 2 TdT-Fluor in situ apoptosis detection kit (Trevigen, USA) on NSCs plated in Lab-Tek (USA) chamber slides treated in triplicate wells with concentrations of cytokines as described in earlier in vitro studies on neural cells (Ben Hur et al, 2003; Cacci et al, 2005; Sheng et al, 2005; Belanger et al, 2011): IL-1 $\beta$ (20 ng/ml); TNF- $\alpha$ (20 ng/ml); IFN- $\gamma$ (20 ng/ml); IL-6 (50 ng/ml), IL-10 (20 ng/ml), IL-4 (20 ng/ml), TGF- $\beta(10 \mathrm{ng} / \mathrm{ml})$ and combinations thereof, for a period of 48 or 72 hours. Nuclei were counterstained with DAPI (Sigma Aldrich, USA). 79 high power fields per well of the chamber slides were counted to a total of 300-400 cells per well. For the MTT (3-4, 5-dimethylthiazol-2-yl-2,5diphenyltetrazolium bromide) reduction assay for cell viability/survival, NSCs were plated in 96-well tissue culture plates and treated with cytokines, with 6 wells per condition. At the end of each experiment medium from each chamber-slide well was removed and 100 $\mu \mathrm{l}$ MTT reagent $(0.5 \mathrm{mg} / \mathrm{ml}$ in DMEM $)$ added in the dark and plates incubated overnight in the $\mathrm{CO}_{2}$ incubator at $37 \mathrm{C}$. The reaction was then stopped by addition of $100 \mu \mathrm{l} /$ well of $10 \%$ SDS- $0.01 \mathrm{~N} \mathrm{HCl}$ and kept in the dark for 6 hours on a slow shaker. The supernatant from each well was analyzed spectrophotometrically using a TECAN ELISA plate reader (Switzerland) at $570 \mathrm{~nm}$ wavelength. Readings from the different conditions were expressed as percent viability with respect to control. Statistical analysis was performed by one-way ANOVA followed by post-hoc Tukey-Kramer tests.

\section{Results and Discussion}

A typical Raman spectrum of an NSC is shown in Fig. 2 with Raman frequencies assigned to the multifarious peaks that are clearly resolved. We utilized available literature to carry out the frequency assignment (Bankapur et al., 2010; Parker, 1983). 


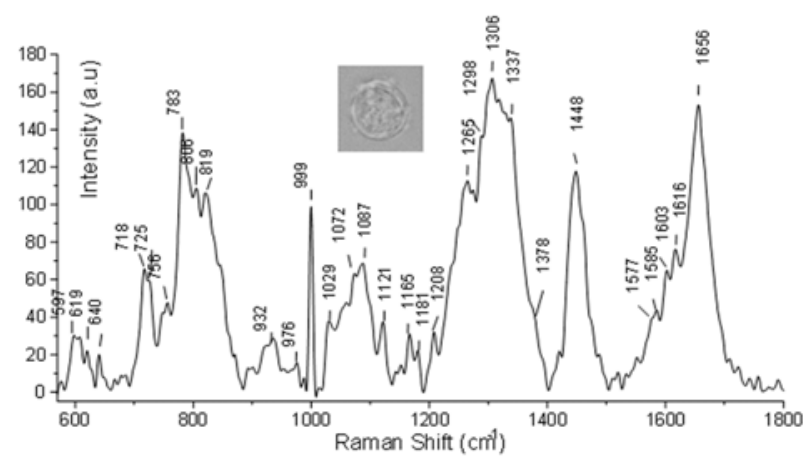

Fig. 2: Typical Raman spectrum of an optically trapped neural stem cell (inset) recorded with $25 \mathrm{~mW}$ of laser power and 60 sec of acquisition time while giving 5 average accumulations

Table 2 lists the assigned frequencies for the peaks in our spectra; the tabulated data provide an overview of the spectral features that characterize the stem cells used in our experiments. Although a detailed analysis of the individual Raman features is not within the scope of the present report, we note that the Raman spectrum is dominated by lines that correspond to signatures expected from proteins, lipids, and nucleic acids - the major building blocks of cells.

For the purposes of the present set of experiments, we focus on those parts of the spectrum that show differences before and after addition to the cell suspension of the inflammatory cytokines, TNF$\alpha$ and IFN- $\gamma$, and anti-inflammatory cytokines IL10 . We find that these differences mainly manifest themselves in the region of $783 \mathrm{~cm}^{-1}$, a spectral line that is primarily ascribed to DNA (Table 2). Figures 3-5 show typical spectra we have measured for different cells. Differences (percentage increase or decrease) in spectral intensity of the DNA peak post cytokine treatment are clearly brought out in Figs. 3D, 4D, and 5D (which show post-treatment spectra with the respective control spectra subtracted). For clarity of presentation we have divided cells into two groups, depending on whether the resulting intensity of the DNA peak is high or low relative to that in the respective baseline control spectrum.

Figs. 3D,4D show difference spectra corresponding to Group 1 (increase in intensity of the DNA band); Fig. 5D shows difference spectra
Table 2: Assignment of peaks observed in the Raman spectrum shown in Figure 2

\begin{tabular}{|c|c|c|}
\hline & $\begin{array}{l}\text { Frequency } \\
\left(\mathrm{cm}^{-1}\right)\end{array}$ & Assignments \\
\hline 1 & 597 & p: Amide VI (membrane) \\
\hline 2 & 619 & Phe: $\mathrm{C}-\mathrm{C}$ twist \\
\hline 3 & 640 & p: C-S str, Tyr: C-C twist \\
\hline 4 & 718 & Phospholipid:Choline: C-N symstr \\
\hline 5 & 725 & A \\
\hline 6 & 756 & $\mathrm{~T}$, Trp \\
\hline 7 & 783 & C,T,U, DNA or Lip:O-P-O diester sym str \\
\hline 8 & 806 & DNA:bk:O-P-O sym str \\
\hline 9 & 819 & Phospholipid: O-P-O diesterantisymstr \\
\hline 10 & 932 & p: skeletal vibration \\
\hline 11 & 976 & Deoxyribose \\
\hline 12 & 999 & Phe: C-C skeletal vibration \\
\hline 13 & 1029 & Phe, p: C-N str \\
\hline 14 & 1072 & Phospholipid: C-O str \\
\hline 15 & 1087 & $\begin{array}{l}\text { DNA:bk:O-P-O sym str, p:C-N str, } \\
\text { Phophoryl Cholin: } \mathrm{PO}_{3}^{-2} \text { anti-sym str }\end{array}$ \\
\hline 16 & 1121 & p: C-N str \\
\hline 17 & 1165 & Collagen: $\mathrm{NH}_{3}^{+}$ \\
\hline 18 & 1181 & $\begin{array}{l}\text { Tyr, NA base external C-N Str, Phe, p:C-H } \\
\text { bend }\end{array}$ \\
\hline 19 & 1208 & Tyr, Phe, A, T, p: Amide III \\
\hline 20 & 1265 & p: Amide III, C, A \\
\hline 21 & 1298 & p: Amide III \\
\hline 22 & 1306 & A, p: Amide III,Phospholipid: $\mathrm{CH}_{2}$ \\
\hline 23 & 1337 & A, G, p: C-H def \\
\hline 24 & 1378 & $\mathrm{~T}, \mathrm{~A}, \mathrm{G}$ \\
\hline 25 & 1448 & $\mathrm{p}: \mathrm{C}-\mathrm{H}_{2}$ def \\
\hline 26 & 1577 & $\mathrm{~A}, \mathrm{G}$ \\
\hline 27 & 1585 & $\mathrm{~A}, \mathrm{G}$ \\
\hline 28 & 1603 & Phe, Tyr, p: C=C \\
\hline 29 & 1616 & Tyr, Trp, p: C=C \\
\hline 30 & 1656 & p: Amide I \\
\hline
\end{tabular}

Abbreviations: def: deformation, bk: vibration of DNA backbone, Str: stretching, U,C,T,A,G:ring breathing modes of the DNA/ RNA bases, Tyr: Tyrosine, Trp: Tryptophan, Phe: Phenylalanine, p: Protein, Lip: Lipid Rib: ribose, NA: nucleic acids 

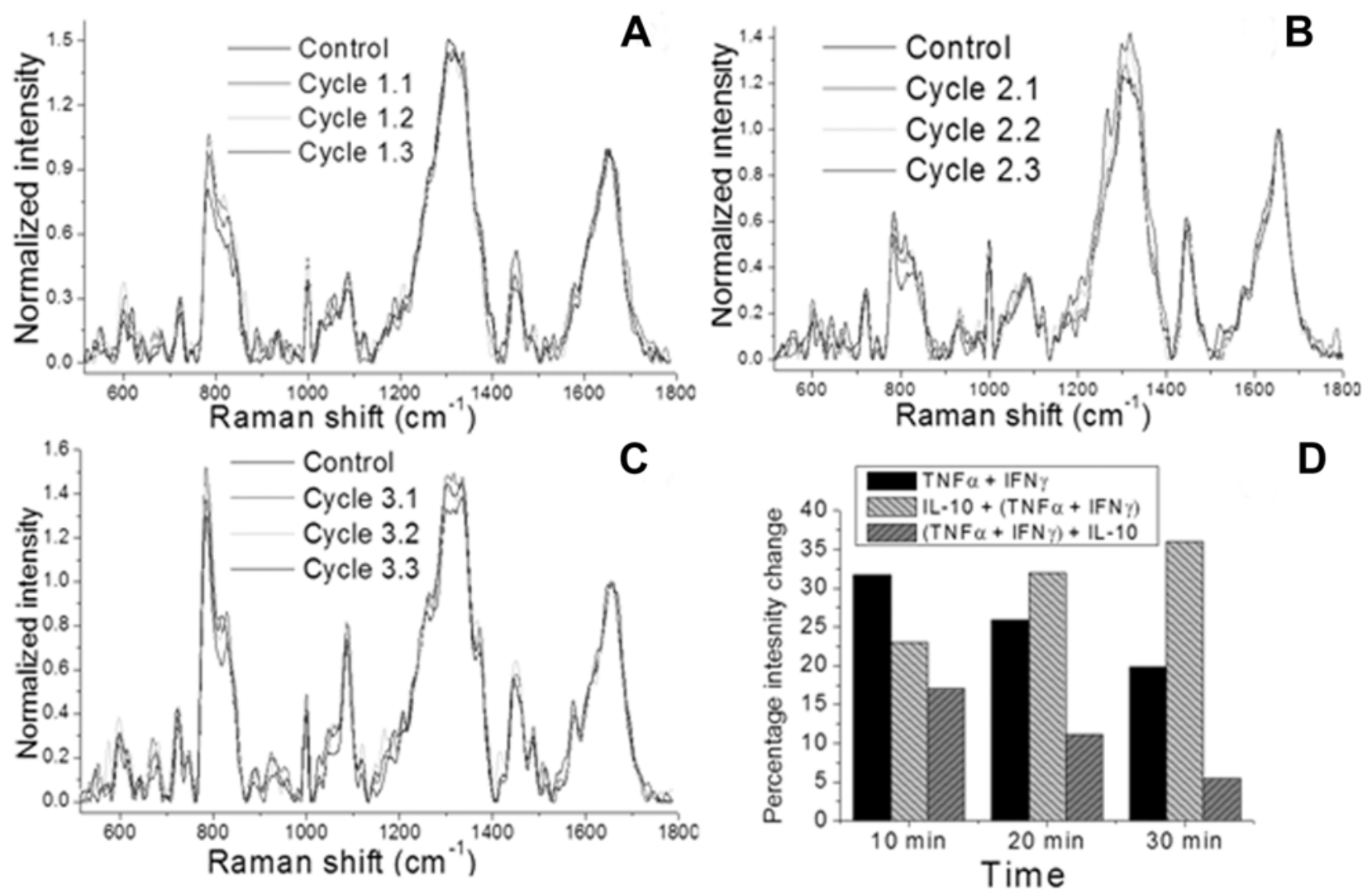

Fig. 3: A-C: Overlaid Raman spectra of different Group I NSCs (numbered 1, 2, 3 - see text) before and after cytokine treatments 1: TNF- $\alpha$ + IFN- $\gamma$; 2: IL-10 + (TNF- $\alpha$ and IFN- $\gamma)$; and 3: (TNF- $\alpha$ and IFN- $\gamma)+$ IL-10. D: Percentage differences in the intensity of the $783 \mathrm{~cm}^{-1}$ DNA peak under the three cytokine treatments. Note the high $(30 \%)$ increase in intensity just 10 minutes after addition of (TNF- $\alpha+I F N-\gamma)$ and the reversal upon addition of IL-10 either 10 minutes prior or 10 minutes after treatment with (TNF- $\alpha+$ IFN- $\gamma)$

corresponding to Group II (decrease in intensity of the DNA band). Interestingly, we find that the response of the cells to the added cytokines depends on whether they are Group I or Group II cells.In group I cells (Figs. 3D) the addition of TNF- $\alpha+$ IFN- $\gamma$ produces a high-intensity DNA band at 10 minutes which reduces significantly (from $32 \%$ to $\sim 20 \%$ ) at 30 minutes. Pre-treatment with IL-10 (prior to addition of TNF- $\alpha+$ IFN- $\gamma$ ) reverses this trend (the enhancement being from $\sim 23 \%$ to $\sim 34 \%$ ). On the other hand, post-treatment with IL-10 produces the same trend as treatment with TNF- $\alpha+$ IFN- $\gamma$, albeit at reduced levels. These trends are qualitatively reproduced in another set of Group I NSCs (Fig. 4D). In Group II cells, on the other hand, the initial TNF- $\alpha$ + IFN- $\gamma$ treatment resulted in a marked decrease in the intensity of the DNA band at 10 minutes. The extent of such decrease is seen to diminish at 20 minutes and 30 minutes (from $-15 \%$ to $-4.5 \%$ ). Here also, pre-treatment with IL-10 reverses this trend (from $-12 \%$ at 10 minutes to $-18 \%$ at 30 minutes). Post-treatment with IL-10 again produces the same effect as in the case of Group I NSCs.

There is an acute paucity of earlier work with which we can compare our present observations. Apoptosis has commonly been described as occurring in a series of distinct, morphological and biochemical stages (Hacker, 2000; Saraste and Pulkki, 2000). The initial stages involve activation of intracellular pathways and only later are the characteristic morphological features such structural changes in DNA, nuclear shrinkage, chromatin and cytoplasmic condensation and disintegration evident. Nuclear changes play a predominant role in descriptions of apoptosis with early chromatin condensation first along 

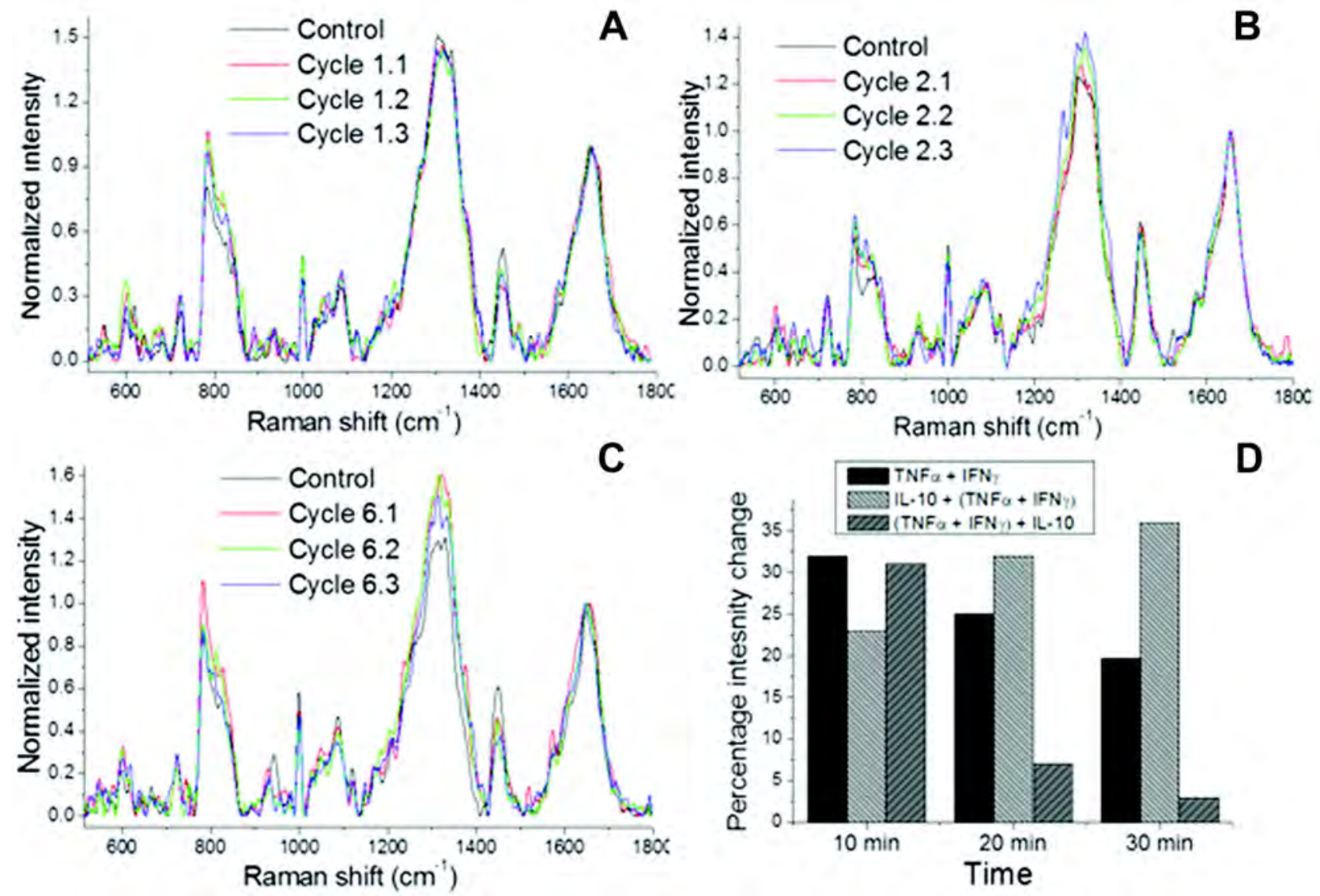

Fig. 4: A-C: Overlaid Raman spectra of different Group I NSCs (numbered 1, 2, 6 - see text) before and after cytokine treatments 1: TNF- $\alpha+$ IFN- $\gamma$; 2: IL-10 + (TNF- $\alpha$ and IFN- $\gamma)$; and 3: (TNF- $\alpha$ and IFN- $\gamma)+$ IL-10. D: Percentage differences in the intensity of the $783 \mathrm{~cm}^{-1}$ DNA peak under the three cytokine treatments

the nuclear membrane. A study of early, pre-apoptotic nuclei described such changes (Johnson et al., 2000). Chromatin condensation involving the entire nucleus and nuclear degradation with fragmentation, considered by some to be the hallmark of apoptosis, occurred at a later stage, along with phosphatidylserine externalization and formation of apoptotic bodies. The molecular events responsible for these are, as yet, not completely understood.

Although there are no spectroscopic studies that have looked at apoptosis at early time points (on the scale of minutes, as in our study), there have been experiments that have probed changes of apoptosis in live cells on long times scales, between 2 and 72 hours (Zoladek et al., 2011; Uzunbajakava et al., 2003; Verrier et al., 2004; Ong et al., 2012; Krafft et al., 2006; Moritz et al., 2010). These have yielded results that are contradictory, especially with regards to the intensity of the DNA spectral band in the region $774 \mathrm{~cm}^{-1}$ to $805 \mathrm{~cm}^{-1}$ (with a peak at $\sim 783 \mathrm{~cm}^{-1}$ ). Zoladek et al. (2011) studied human breast cancer cells and showed a $\sim 1.5$ fold increased intensity of Raman bands associated with DNA in all cells exposed to 6 hours of etoposide; this increase in intensity was assigned to nuclear condensation seen in the early stages of apoptosis. Intensity increase of the same Raman band was also reported for apoptotic HeLa (Uzunbajakava et al., 2003), but here the increase was much higher, a factor of 4-5. On the other hand, decreased intensity of the DNA band in apoptotic cells has been reported in other studies (Verrier et al., 2004; Ong et al., 2012; Krafft et al., 2006). Such decrease was attributed to either marked nuclear condensation (pyknosis) or to the cleavage of DNA into short fragments packed into apoptotic bodies. Krafft et al. (2006) have suggested that 

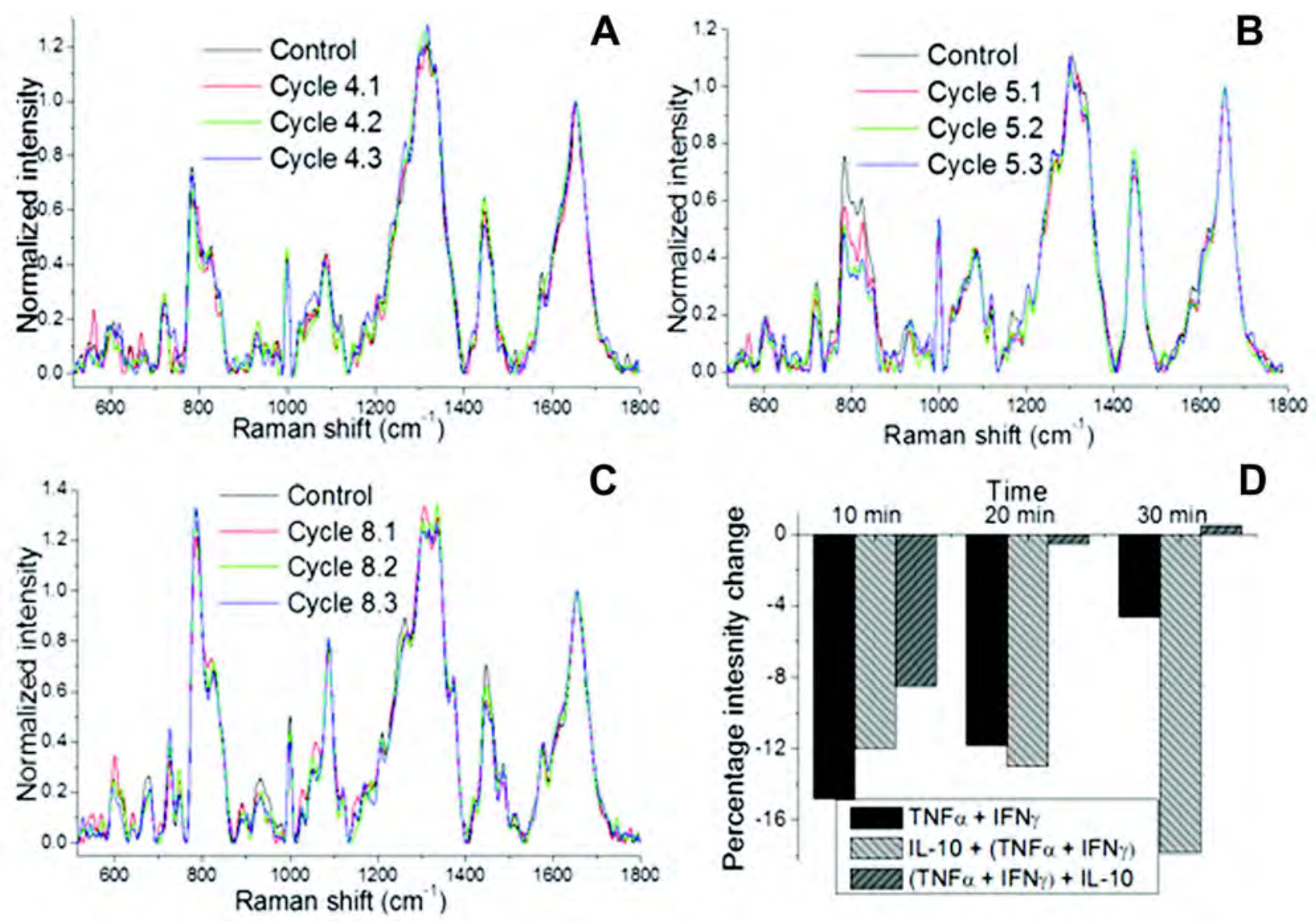

Fig. 5: A-C: Overlaid Raman spectra of different Group II NSCs (numbered 4, 5, 8 - see text) before and after cytokine treatments 1: TNF- $\alpha$ + IFN- $\gamma$; 2: IL-10 + (TNF- $\alpha$ and IFN- $\gamma)$; and 3: (TNF- $\alpha$ and IFN- $\gamma)+$ IL-10. D: Percentage differences in the intensity of the $783 \mathrm{~cm}^{-1}$ DNA peak under the three cytokine treatments. Note the marked decrease (by $\left.15 \%\right)$ of the intensity of the DNA peak just 10 minutes after addition of (TNF- $\alpha+$ IFN- $\gamma$ ), which is further accentuated (to 18\%) by IL-10 pre-treatment at 30 minute

increased condensation might lead to more base-base interaction and consequently, to decreased DNA intensities. In only one earlier study (Moritz et al., 2010) has it been reported that the DNA band both increased as well as decreased in intensity. These experiments were conducted on Jurkat cells, with spectral measurements made on time scales of 12-72 hours. Here again the increase in Raman signal in some cells was attributed to chromatin condensation and nuclear fragmentation. The decrease in intensity in another group of cells was, on the other hand, attributed to late-stage apoptosis where nuclear fragments were expelled from the cell, leaving behind an "empty" cell.

As already noted, our spectroscopic results on very early-stage apoptosis also show that cells can be divided into two groups: those that show an increase in the intensity of the DNA band and those that show the opposite. These changes were seen in IFN- $\gamma$ and TNF- $\alpha$ - treated NSCs at as early a stage as 10-30 minutes; interestingly, these changes were seen by us to be partially reversed upon addition of IL-10 either 10 minutes before or 10 minutes after treatment with IFN- $\gamma$ and TNF- $\alpha$. Moreover, these changes were not accompanied by any discernible morphological alterations of apoptosis in all the cells we studied. Neither is there corresponding information in the literature on detectable morphological changes at such early time scales. We postulate, as in the case of prior work, that the increasing DNA intensity may be due to early nuclear condensation, whereas the decreased DNA intensity could be indicative of the occurrence 
of cavitation between the clumped chromatin in the "pro-apoptotic" stage described by Johnson et al. (2000). This variability in response of the different NSCs in our study may be attributed to asynchrony of cell cycles of the NSCs.

The application of optical trapping methods on biological matter inevitably raises the important question of potential laser-induced damage within the trapping volume. Such potential damage is expected to be dependent on both wavelength and the incident laser power levels used. It might be expected that photochemically induced alterations in the biochemical properties of a trapped cell might include damage to DNA, alterations in cell metabolism, and the onset of chemical processes like oxidation (Calmettes and Berns, 1983). The tight spatial focusing that underpins optical trapping inevitably generates localized laser intensities that might easily exceed several $\mathrm{MW} \mathrm{cm}^{-2}$ within the focal volume, resulting in localized thermal effects like the formation of bubbles (Ramanandan et al., 2009), the creation of thermal traps, and generation of acoustic waves (Lapotko and Zharov, 2005). Any or all of these effects might be expected to give rise to photomechanical effects that might prove to be deleterious to the trapped stem cells of interest in our study. We refer to a cogent discussion of such experimental considerations (Snook et al., 2009) in the light of which we have undertaken the experimental design of our Raman Tweezers apparatus and our measurement scheme.

Firstly, laser power levels used to trap our cells, and to record Raman spectra from individual trapped cells, were measured just after the microscope objective in our experimental set-up by means of an integrating sphere coupled to a photodiode. Laser power levels incident on any trapped cell were kept low enough to ensure cell viability. In earlier experiments, we have made measurements on trapped cells for periods as long as $\sim 1$ hour, recording Raman spectra every 5-10 minutes, so as to ensure that recorded spectra were consistent during the entire stretch of each experimental run to minimize concerns regarding cell viability. Optical trap work on live cells that has been conducted in our laboratory over several years (see Bankapur et al., 2010; Zachariah et al.,
2010; and references therein) has yielded evidence that occurrence of optically-induced damage is unlikely when incident laser power levels are kept less than $\sim 30 \mathrm{~mW}$ as long as the irradiated cells are kept in a physiologically relevant medium, which they were in our present series of measurements. The lowest possible incident power levels were utilized that we found to we commensurate with adequate signal-tonoise ratio and spectral resolution.

Secondly, our choice of laser wavelength was dictated by the following considerations. The efficiency of Raman scattering is known to exhibit a wavelength dependence that scales as $\lambda^{-4}$. This indicates the desirability of using short wavelengths. However, earlier work has established that as indicated by the work of Neuman et al. (1999), shorter wavelengths result in increased propensity for laserinduced photo damage. The use of longer wavelengths also offers a potential advantage of reducing fluorescence effects that compete with the weak Raman signals. However, longer wavelengths lead to inescapable problems related to the efficiency of CCD-based photon detectors that are readily available at present. As discussed by Snook et al. (2009), the best available detection efficiency of $\sim 50 \%$ is obtained over the wavelength range $600-800 \mathrm{~nm}$. The corresponding value around $1 \mu \mathrm{m}$ wavelength is $10 \%$ while at $1064 \mathrm{~nm}$ it is almost zero. In the present series of experiments we took care to measure Raman spectra of untreated NSCs at time intervals of 10, 20, and 30 minutes. We found no discernible differences in spectral lines profiles but intensities showed insignificant variability, to the extent of $+2 \%$ and $5 \%$. These small variabilities confirm that there is no laser-induced damage to our NSCs.

We used the TUNEL assay to quantitatively screen for cell death of NSCs exposed to various pro- and anti-inflammatory cytokines in vitro. From our results (Fig. 6A) it is evident that concomitant addition of IFN- $\gamma$ and TNF- $\alpha$ caused a significant amount of NSC apoptosis ( $\mathrm{p}<0.001$ )-greater than that seen with either cytokine added singly or with any of the other pro-inflammatory cytokines that we tested. When time-course experiments were performed with IFN- $\gamma$ and TNF- $\alpha$, significant 
A

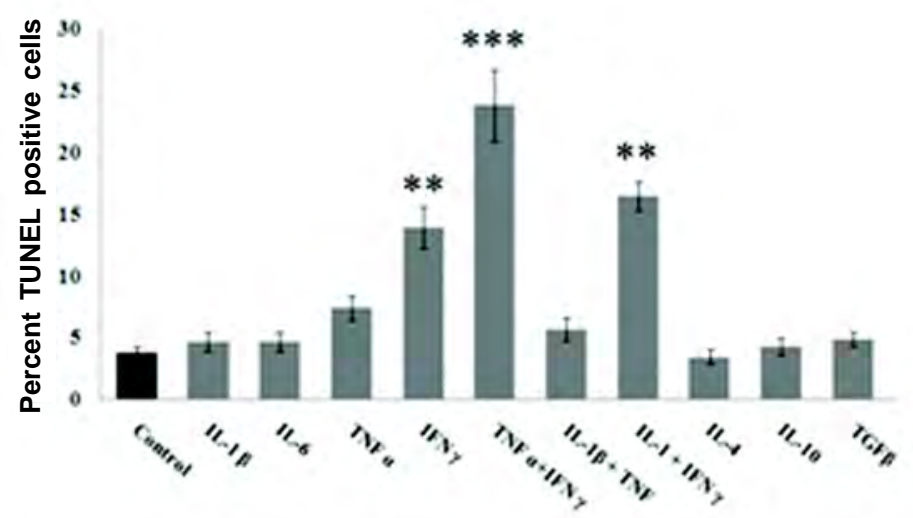

C

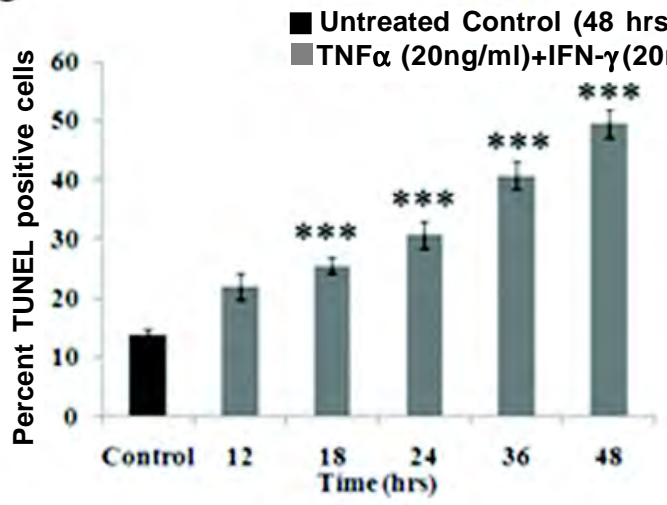

E

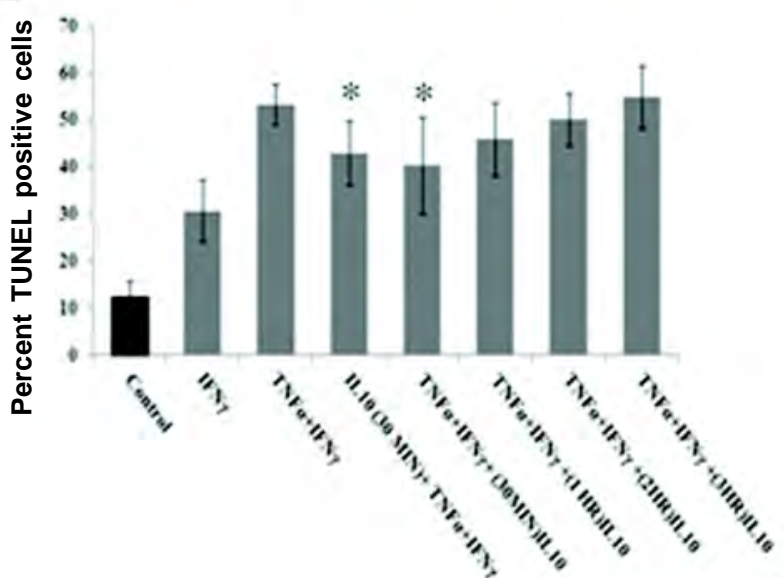

B

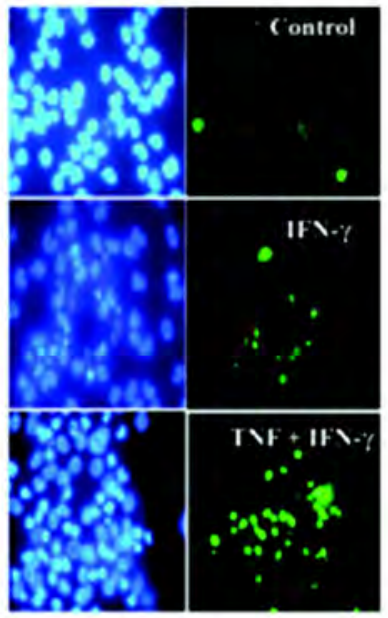

D

Untreated Control (48 hrs)

TNF $\alpha(20 \mathrm{ng} / \mathrm{ml})+\mathrm{IFN}-\gamma(20 \mathrm{ng} / \mathrm{ml})$

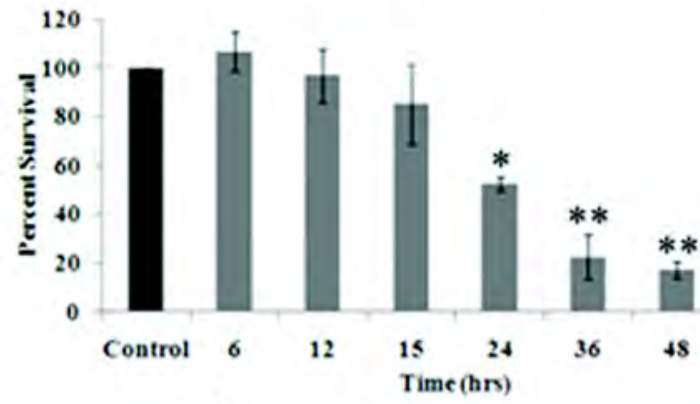

$\mathbf{F}$

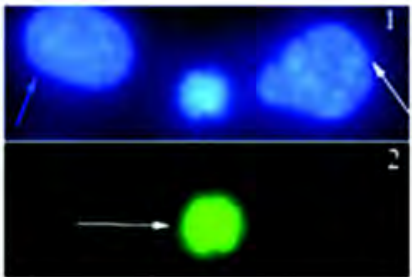

Fig. 6: Pro-inflammatory cytokines IFN- $\gamma$ and TNF- $\alpha$ used concomitantly cause significant apoptosis (p<0.001) of NSCs that can be partly reversed by addition of the anti-inflammatory cytokine IL-10 either 30 min prior or 30 min after treatment with IFN- $\gamma$ and TNF- $\alpha$ in vitro. A: Quantitation of the pro-apoptotic effect of various pro-inflammatory and anti-inflammatory cytokines on NSCs by the TUNEL assay over 72 hours in vitro; B: Morphology of untreated (control), IFN- $\gamma$-treated and IFN- $\gamma+$ TNF- $\alpha$ - treated TUNEL positive NSCs; C-D: Viability of IFN- $\gamma$ and TNF- $\alpha$-treated NSCs over time by the TUNEL and MTT assays, respectively; E: Addition of IL-10 to NSCs 30 min prior, and 30 min, 1 hour, 2 hours, and 3 hours after IFN- $\gamma$ and TNF- $\alpha$ treatment in vitro. The pro-apoptotic effect of IFN- $\gamma$ and TNF- $\alpha$ treatment on NSCs was partly reversed only when IL-10 was added 30 min prior $(p<0.05)$ or 30 min after $(p<0.01)$. F: Details of DAPI-stained (1) and TUNEL-stained (2) nuclei of IFN- $\gamma+$ TNF- $\alpha$-treated NSCs showing an apoptotic TUNEL +ve nucleus, a convoluted nucleus with condensed chromatin (white arrow) and the nucleus of an NSC showing no changes (blue arrow) 
apoptosis was detectable only at 18-24 hours by the TUNEL and MTT assays, respectively (Fig. 6 C,D), in concordance with other studies (Ben-Hur et al., 2003; Cacci et al., 2005). When the anti-inflammatory cytokine IL-10 was added either $30 \mathrm{~min}$ prior to or 30 min after IFN- $\gamma$ and TNF- $\alpha$, we observed partial reduction of this outcome as well as improved viability of the NSCs ( $<<0.05$ and $\mathrm{p}<0.01$, respectively) (Fig. 6E). This was not evident when IL-10 was added later, at 1 hour, 2 hours, or 3 hours posttreatment (Fig. 6E). Until recently apoptosis was considered to be an irreversible process, especially once the critical checkpoints such as mitochondrial fragmentation and DNA damage occurred. In contrast, a few recent studies have provided indications that apoptosis may be reversible either in the early stages (Geske et al., 2001) or even in late stages (Tang et al., 2012).

It is noteworthy that, in our experiments, although changes were not visually discernable at very early time points at which spectroscopic changes were observed, various morphological stages of apoptosis were, indeed, evident in the TUNEL assay, as shown in Fig. 6F.

Neural stem cells have been reported to express receptors for both IFN- $\gamma$ and TNF- $\alpha$ (TNF-RI, TNFRII) but not IL-1 or IL-6 (Ben-Hur et al., 2003; Iosif et al., 2006). Ben-Hur et al. (2003) reported a decrease in the proliferation of NSCs isolated from newborn rat striata, when both IFN- $\gamma$ and TNF- $\alpha$ were added together, whereas IFN- $\gamma$ alone caused increased apoptosis that was partially blocked by the addition of TNF- $\alpha$. Other studies have reported that TNF- $\alpha$ caused increased apoptosis when added to cells of a neural stem cell line (Cacci et al., 2005) or in fetal neural stem cells through TNF-RI (Sheng et al., 2005), or resulted in cell death of NSCs by necrosis (Wong et al., 2008). IFN- $\gamma$ inhibited NSC proliferation and differentiated NSCs but did not cause apoptosis in one report (Wong et al., 2004) whereas in another, NSCs and their differentiated progeny were found to express both types of IFN- $\gamma$ receptors and IFN- $\gamma$ treatment of NSCs resulted in the development of dysfunctional differentiated progeny (Walter et al., 2011). These apparently conflicting results, including our finding of a pro-apoptotic effect of IFN- $\gamma$ as well as the additive effect of concomitant IFN- $\gamma$ and TNF$\alpha$, could possibly be an outcome of cells in these studies being isolated from differing species of different ages, from different regions (that is, subventricular zone or subgranular zone of the hippocampus) and whether the experiments were conducted in vitro or in vivo. It general, it is accepted that suppressing the effects of IFN- $\gamma$ and TNF- $\alpha$ is beneficial in improving stem cell therapies (Breton and Mao-Draayer, 2011). The anti-inflammatory effects of IL-10 have only recently been studied. IL10 is also increased in neuro-inflammation (Csuka et al., 1999; Hensler et al., 2000; Dziurdzik et al., 2004; Shiozaki et al., 2005; Kamm et al., 2006; Kirchoff et $a l ., 2008$ ), and counteracts the deleterious effects of pro-inflammatory cytokines such as IFN- $\gamma$ and IL17 (Knoblach and Faden, 1998; Yang et al., 2009). IL-10 increases both neurogenes is and oligodendrogenesis in vivo (Yang et al., 2009). More recently, adeno-associated virus-mediated overexpression of neuronal IL-10 ameliorated cognitive dysfunction in a mouse model of Alzheimer's disease (Kiyota et al., 2012) and EAE, the animal model of MS, was suppressed when IL-10-transduced NSCs were injected intravenously (Klose et al., 2013). When IL-10 was administered by different routes in a rodent model of TBI post-injury (Knoblach and Faden, 1998), the outcome was improved only by systemic administration in vivo but not when IL-10 was given intra-cerebroventricularly. Our findings show that IL-10 has a direct anti-inflammatory effect in counteracting the pro-apoptotic effects of proinflammatory cytokines, IFN- $\gamma$ and TNF- $\alpha$, on NSCs in vitro. Interleukin-10 has powerful immuneregulatory properties and recombinant IL-10 has been tried for the therapy of established immune diseases with limited success, warranting further investigation (Assadullah et al., 2003).

It is pertinent to note that the primary significance of our findings lies in the recognition that inflammationinduced damage in neural stem cells can occur extremely rapidly and that it can be reversed by early anti-inflammatory measures, such as IL-10 in our study. In acute inflammatory conditions, such as TBI, the inflammatory response begins within minutes - 
much earlier than was previously thought, and this is accompanied by an increase in levels of many of the pro- and anti-inflammatory cytokines (Kamm et al., 2006; Frugier et al., 2010) thus leaving a narrow window for endogenous or exogenous interventions to prevent significant damage to neural stem/

\section{References}

Ashkin A (1970) Acceleration and trapping of particles by radiation pressure Phys Rev Letters 24 156-159

Assadullah K, Sterry W and Volk H D (2003) Interleukin-10 therapy- review of a new approach Pharmac Rev 55 241269

Bankapur A, Zachariah E, Chidangil S, Valiathan M and Mathur D (2010) Raman tweezers spectroscopy of live, single red and white blood cells PLOS ONE 5 pe10427

Belanger M, Allaman I and Magistretti P J (2011) Differential effects of pro- and anti-inflammatory cytokines alone or in combination on the metabolic profile of astrocytes $J$ Neurochem 116 564-576

Ben-Hur T, Ben-Menachem O, Furer V, Einstein O, MizrachiKol R and Grigoriadis N (2003) Effects of proinflammatory cytokines on the growth, fate and motility of multipotential neural precursor cells Mol Cell Neurosci 24 623-631

Breton J and Mao-Draayer Y (2011) Impact of cytokines on neural stem/progenitor cell fate $J$ Neurol Neurophysiol $\mathbf{S 4}$ 01-012

Cacci E, Classen J H and Kokaia Z (2005) Microglia-derived tumour necrosis factor- $\alpha$ exaggerates death of newborn hippocampal progenitor cells in vitro J Neurosci Res $\mathbf{8 0}$ 789-797

Calmettes P P and Berns M W (1983) Laser-induced multi-photon processes in living cells Proc Natl Acad Sci USA 807197 7199

Christie K J and Turnley A M (2013) Regulation of endogenous neural stem/progenitor cells for neural repair - factors that promote neurogenesis and gliogensis in the normal and damaged brain Front Cell Neurosci 6 1-18

Conti A C, Raghupathi R, Trojanowski J Q and McIntosh T K (1998) Experimental brain injury induces regionally distinct apoptosis during the acute and delayed post-traumatic period J Neurosci 18 5663-5672

Csuka E, Morganti-Kossman M C, Lenzlinger P M, Joller H, Trentz O and Kossman T (1999) IL-10 levels in cerebrospinal fluid and serum of patients with severe traumatic brain injury: relationship to IL-6, TNF- $\alpha$, TGF$\beta$ and blood brain barrier function $J$ Neuroimmunol 101 progenitors of the hippocampus. Further investigations, especially in animal models, are required to verify these findings in vivo as well as on the prognosis of clinical symptoms secondary to neuro-inflammation, such as cognitive deficits.

\section{1-221}

Dalgard C L, Cole J T, Kean W S, Lucky J J, Sukumar G, McMullen D C, Pollard H B and Watson W D (2012) The cytokine temporal profile in rat cortex after controlled cortical impact Front Mol Neurosci 5 1-10

Dziurdzik P, Krawczyk L, Jalowiecki P, Kondera-Anasz Z and Menon L (2004) Serum interleukin-10 in ICU patients with severe acute central nervous system injuries Inflamm Res 53 338-343

Frugier T, Morganti-Kossman M C, R'Reilly D and McLean C A (2010) In situ detection of imflammatory mediators in post mortem human brain tissue after traumatic injury $J$ Neurotrauma 27 497-507

Geske F J, Lieberman R, Strange R and Gerschenson L E (2001) Early stages of p53-induced apoptosis are reversible Cell Death Diff 8 182-191

Goodman J C, Van M, Gopinath S P and Robertson C S (2008) Pro-inflammatory and pro-apoptotic elements of the neuro-inflammatory response are activated in traumatic brain injury Acta Neurochir Suppl 102 437-439

Hacker G (2000) The morphology of apoptosis Cell Tissue Res 301 5-17

Hensler T, Sauerland S, Riess P, Hess S, Helling H J, Andermahr J, Bouillon B and Neugebauer E A (2000) The effect of additional brain injury on systemic interleukin-10 and inteleukin-13 levels in trauma patients Inflamm Res 49 524-528

Holmin S and Hojeberg B (2004) In situ detection of intracerebral cytokine expression after human brain contusion Neurosci Lett 369 108-114

Iosif R E, Ekdahl C T, Ahlenius H, Pronk C J H, Bonde S, Kokaia Z, Jacobsen S W and Lindvall O (2006) Tumour necrosis factor receptor 1 is a negative regulator of progenitor proliferation in adult hippocampal neurogenesis $J$ Neurosci 26 9703-9712

Johnson V L, Ko S C W, Holmstron T H, Eriksson J E and Chow S C (2000) Effector caspases are dispensible for the early nuclear morphological changes during chemical-induced apoptosis J Cell Sci 113 2941-2953

Kamm K, Vanderkolk W, Lawrence C, Jonker M and Davis A T 
(2006) The effect of traumatic brain injury upon the concentration and expression of interleukin-1 $\beta$ and interleukin-10 in the rat $J$ Trauma 60 152-157

Kerr J F R, Wyllie A H and Curie A R (1972) Apoptosis: a basic biological phenomenon with wide-range implications in tissue kinetics Br J Cancer 26 239-257

Kirchoff C, Buhmann S, Bogner V, Stegmaier J, Leidel B A, Braunstein V, Mutschler W and Biberthaler P (2008) Cerebrospinal IL-10 concentration is elevated in nonsurvivors as compared to survivors after severe traumatic brain injury Eur J Med Res 13 464-468

Kiyota T, Ingraham K L, Swan R J, Jacobsen M T, Andrews S J and Ikezu T (2012) AAV serotype-2/1-mediated gene delivery of anti-inflammatory interleukin-10 enhances neurogenesis and cognitive function in $\mathrm{APP}+\mathrm{PS} 1$ mice Gene Ther 19 724-733

Klose J, Schmidt N O, Melms A, Dohi M, Miyazaki J I, Bischof F and Greve B (2013) Suppression of experimental allergic encephalomyelitis by interleukin-10 transduced neural stem/progenitor cells J Neuroinflamm 10117

Knoblach S and Faden AI (1998) Interleukin-10 improves outcome and alters proinflammatory cytokine expression after experimental traumatic brain injury Exp Neurol 153 143151

Krafft C, Knetschke T, Funk R H W and Salzer R (2006) Studies on stress-induced changes at the subcellular level by Raman microspectroscopic mapping Anal Chem 78 4424-4429

Krawohl M D and Kaiser J L (2004a) Chemokines promote quiescence and survival of human neural progenitor cells Stem Cells 22 109-118

Krawohl M D and Kaiser J L (2004b) HIV-1 promotes quiescence in human neural progenitor cells J Infect Dis 190 216-226

Lapotko D O and Zharov V P (2005) Lasers Surg Med 36 22-30

Matthews Q, Brolo A G, Lum J, Duan X and Jirasek A (2011) Raman spectroscopy of single human tumour cells exposed to ionizing radiation in vitro Phys Med Biol 56 19-38

Morganti-Kossman M C, Rancan M, Otto V L, Stahel P F and Kossman T (2001) Role of cerebral inflammation after traumatic brain injury: a revisited concept Shock $\mathbf{1 6} 165$ 177

Moritz T J, Taylor D S, Krol D M, Fritch J and Chan J W (2010) Detection of doxorubicin-induced apoptosis of leukemic T-lymphocytes by laser tweezers Raman spectroscopy Biomed Opt Express 1 1138-1147

Neuman K, Chadd E H, Liou G F, Bergman K and Block S M (1999) Characterization of photodamage to Escherichia coli in optical traps Biophys $J 77$ 2856-2863

Ong Y H, Lim M and Liu Q (2012) Comparison of principal component analysis and biochemical component analysis in Raman spectroscopy for the discrimination of apoptosis and necrosis in K562 leukemia cells Optics Exp 2022158 22171

Palmer T D, Takahashi J and Gage F (1997) The adult rat hippocampus contains primordial neural stem cells $\mathrm{Mol}$ Cell Neurosci 8 389-404

Parker F S (1983) Applications of infrared, Raman and resonance Raman spectroscopy in biochemistry, Springer-Verlag

Peng H, Whitney N, Wu Y, Tian C, Dou H, Zhou Y and Zheng J (2008a) HIV-1-infected and/or immune-activated macrophage-secreted TNF-alpha effects human fetal cortical neural progenitor cell proliferation and differentiation Glia 56 903-916

Ramanandan G, Dharmadhikari A K, Dharmadhikari J A, Ramachandran H and Mathur D (2009) Bright visible emission from carbon nanotubes spatially constrained on a micro-bubble Opt Exp 17 9614-9620

Sailer K (1996) Radiation-induced structural modifications in dsDNA analysed by FT-Raman spectroscopy Int $J$ Rad Biol 69 601-613

Saraste A and Pulkki K (2000) Morphologic and biochemical hallmarks of apoptosis Cardiovascular Res 45 528-537

Sheng W S, Hu S, Ni T H, Rowen T N, Lokensgard J R and Peterson P K (2005) TNF- $\alpha$ - induced chemokine production and apoptosis in human neural precursor cells J Leuk Biol 78 1233-1241

Shiozaki T, Hayakata T, Tasakai O, Hosotubo H, Fujita K, Mouri T, Tajima G, Kajino K, Nakae H, Tanaka H, Shimazu T and Sugimoto H (2005) Cerebrospinal fluid concentrations of anti-inflammatory mediators in early-phase severe traumatic brain injury Shock $\mathbf{2 3} 406-410$

Snook R D, Harvey T J, Faria C J and Gardner P (2009) Raman tweezers and their application to the study of singly trapped eukaryotic cells Integr Biol 1 43-52

Tang H L, Tang H M, Mak K H, Wang S S, Wong K M, Wong C ST, Wu H Y, Law H T, Liu K, Talbot C C, Lau W K, Montell D J and Fung M C (2012) Cell survival, DNA damage and oncogenic transformation after a transient and reversible apoptotic response Mol Bio Cell 23 2240-2252

Tzong-Shine Y, Zhang G, Liebl D J and Kernie S G (2008) Traumatic brain injury-induced hippocampal neurogenesis requires activation of early nestin-expressing progenitors J Neurosci 28 12901-12912

Uzunbajakava N, Lenferink A, Kraan Y, Volokhina E, Vrensen G, Greve J and Otto C (2003) Nonresonant confocal Raman imaging of DNA and protein distribution in apoptotic cells Biophys J 84 3968-3981 
Verrier S, Notingher I, Polak J M and Hench L L (2004) In situ monitoring of cell death using Raman microspecroscopy Biopolymers 74 157-162

Waldau B and Shetty A K (2008) Behaviour of neural stem cells in the Alzheimer brain Cell Mol Life Sci 65 2372-2384

Walter J, Honsek S D, Illes S, Wellen J M, Hartung H P, Rose C $\mathrm{R}$ and Dihne $\mathrm{M}$ (2011) A new role for interferon gamma in neural stem/precursor cell dysregulation Mol Neurodegen 6 18-30

Whitney N P, Eidem T M, Peng H, Huang Y and Zheng J C (2009) Inflammation mediates varying effects in neurogenesis: relevance to the pathogenesis of brain injury and neurodegenerative disorders $J$ Neurochem 1081343 1359

Wong G, Goldschmit Y and Turnley A M (2004) Interferon- but not TNF- $\gamma$ promotes neural differentiation and neurite outgrowth of murine adult neural stem cells Exp Neurol 187 171-177

Wyllie A H, Kerr J F and Currie A R (1980) Cell death: the significance of apoptosis Int Rev Cytol 68 251-306

Yang L, Lindholm K, Konishi Y, Li R and Shen Y (2002) Target depletion of distinct tumour necrosis factor receptor subtypes reveals hippocampal neuron death and survival through signal transduction pathways J Neurosci 22 30253032

Zachariah E, Bankapur C, Santhosh C, Valiathan M and Mathur D (2010) Probing oxidative stress in single erythrocytes with Raman tweezers J Photochem Photobiol B 110 113116

Zoladek A, Pascut F C, Patel P and Notingher I (2011) Noninvasive time-course imaging of apoptotic cells by confocal Raman micro-spectroscopy J Raman Spect 42 251-258. 